\title{
BIOLOGIA REPRODUTIVA DO CAMARÃO Macrobrachium brasiliense (HELLER, 1862) (CRUSTACEA: DECAPODA: PALAEMONIDAE) EM IGARAPÉS DE TERRA FIRME DA AMAZÔNIA PERUANA
}

\author{
Carmen Rosa GARCíA-DÁVILA ${ }^{1}$, Fernando ALCANTÁRA B. ${ }^{2}$, Elvis \\ VASQUEZ R. ${ }^{3}$, Miquel CHUJANDAMA S. ${ }^{3}$
}

\begin{abstract}
RESUMO - Foi estudada a biologia reprodutiva de Macrobrachium brasiliense (Decapoda: Palaemonidae) com base em 2604 individuos coletados entre outubro de 1994 e agosto de 1995 , em dois igarapés de terra firme da estrada Iquitos-Nauta, Loreto (Amazônia peruana). A proporção sexual fêmea: macho foi de 1,7:1 sendo que as fêmeas foram um pouco mais abundantes que os machos durante todo o periodo de coleta. M. brasiliense apresentou dimorfismo sexual no comprimento cefalotorácico, sendo as fêmeas ligeiramente menores que os machos. A reprodução foi contínua, com o pico reprodutivo entre abril e julho. A fecundidade variou de 15 a 168 ovos por fêmea, observando-se uma relação significativa entre a fecundidade e o comprimento cefalotorácico e o peso. $\mathrm{O}$ volume do ovo não apresentou relação significativa com o comprimento cefalotorácico e a fecundidade.
\end{abstract}

Palavras-chaves: Decapoda, água doce, reprodução, Amazônia, Peru.

Reprodutive Biology of the Shrimp Macrobrachium brasiliense (Heller, 1862) (Crustacea: Decapoda: Palaemonidae) in Small Forest Streams of Peruvian Amazon.

ABSTRACT - The reproductive biology of Macrobrachium brasiliense (Decapoda: Palaemonidae) was studied based on 2,604 specimens collected from October 1994 to August 1995, in two forest streams along the Iquitos-Nauta, highway in Loreto (Peruvian Amazon). The female: male sex ratio was 1.7:1, M. brasiliense showed sexual dimorphism in the cephalotorax length, females being slighthly smaller than males. Reproduction was continuos with a reproductive peak between April and June. Fecundity ranged from 15 to 168 eggs per female. A significant relationship was observed between fecundity and cephalotoraxic length and the weight. Egg volume presented no significant relationship with cephalotoraxic length or fecundity.

Key-worlds: Decapoda, freshwater, reproduction, Amazon, Peru.

\section{INTRODUÇÃO}

A maioria dos estudos sobre camarões amazônicos foram dirigidos a Macrobrachium amazonicum (Heller, 1862), uma vez que é a única espécie nativa pescada artesanalmente na Amazônia (Coelho et al., 1982; Odinetz Collart, 1988; 1992;
Montreuil et al., 1990). Pouco é conhecido sobre os camarões de água doce dos igarapés de terra firme, que adaptaram sua bioecologia a ambientes que apresentam um pulso de inundação mais dinâmico que o dos grandes rios (Walker \& Ferreira, 1985). M. brasiliense (Heller, 1862) é um camarão com ampla distribuição

\footnotetext{
' Programa de Pós-graduação em Biologia de Água Doce e Pesca Interior (BADPI) - Instituto Nacional de Pesquisas da Amazônia (INPA), Caixa postal 478, 69011-970 Manaus, AM.

${ }^{2}$ Instituto de Investigaciones de la Amazonia Peruana- IIAP, Av. Abelardo Quiñones Km 2.5, Aptdo. 784, Iquitos, Loreto - Peru.

${ }^{3}$ Universidad Nacional de la Amazonia Peruana - UNAP, calle Pevas $5^{\text {ta }}$ quadra, Iquitos, Loreto - Peru.
} 
geográfica, ocorrendo nas bacias costeiras do norte de América do Sul, da Venezuela ao Brasil e bacias dos rios Orinoco, Amazonas, São Francisco, Paraguai e Paraná (Coelho \& Ramos-Porto, 1985; López \& Pereira, 1996). Pode ser encontrado tanto na calha dos grandes rios quanto nos lagos, igapós e igarapés de terra firme. Os estudos existentes sobre $M$. brasiliense abrangem principalmente levantamentos faunísticos e taxonomia (Holthuis, 1952; Kensley \& Walker, 1982; Coelho \& Ramos-Porto, 1985; Pereira, 1993; García, 1998), conhecendo-se pouco sobre sua bioecologia. Neste trabalho, foram estudados aspectos da biologia reprodutiva de camarão de água doce M. brasiliense e sua relação com fatores ambientais aquáticos em pequenos igarapés.

\section{MATERIAL E MÉTODOS}

As coletas foram realizadas em dois igarapés de terra firme na estrada Iquitos-Nauta, situados no segmento compreendido entre os povoados de Quistococha ( $3^{\circ} 49,73^{\prime}$ S e $\left.73^{\circ} 19,37^{\prime} \mathrm{W}\right)$ e Allpahuayo ( $3^{\circ} 51^{\prime} \mathrm{S}$ e $\left.73^{\circ} 19,37^{\circ} \mathrm{W}\right)$, nas proximidades de Iquitos (Amazônia peruana) (Fig. 1). As duas estações de coletas caracterizam-se por sofrer pouca influência dos grandes rios e pelo fato do nível das suas águas depender principalmente das precipitações pluviais locais. A profundidade média das estações de coleta foi de $0,50 \mathrm{~m}$, e largura média foi de $2,5 \mathrm{~m}$. $\mathrm{O} \mathrm{pH}$ oscilou entre 5,5 e 6,0 .

As coletas foram quinzenais e realizadas de outubro de 1994 a agosto de 1995. Empregou-se uma rede de mão com a qual era levantada toda a serrapilheira submersa e a vegetação das margens até uma profundidade de $0,3 \mathrm{~m}$. As amostras foram triadas e conservadas em álcool $70 \%$ no local de coleta.

No laboratório foi determinado o sexo de cada individuo com base na presença ou ausência do apêndice masculino no segundo par de pleópodos. A proporção de sexos foi calculada e testada (1:1) com o teste qui-quadrado $\left(\chi^{2}\right)$. A análise morfológica para a identificação da espécie foi realizada em 523 indivíduos adultos, onde contou-se o número de dentes do rostro e mediuse o comprimento cefalotorácico (distância dorsal entre a margem posterior da cavidade orbital e a margem posterior do cefalotórax), em milímetros. As diferenças entre machos e fêmeas foram comparadas com um teste "T". O dimorfismo sexual do segundo par de pereiópodos foi determinado em espécimes adultos medindo-se o apêndice de maior tamanho do par; os dados foram analisados mediante um teste "T". Também foi testada a relação entre o segundo pereiópodo e o comprimento cefalotorácico mediante análise de regressão simples.

$\mathrm{O}$ período reprodutivo foi determinado pela presença de fêmeas ovadas e recém desovadas (identificadas como tais pela presença de resíduos de ovos nos pleópodos e pelas pleuras alongadas). A fecundidade foi determinada pelo 


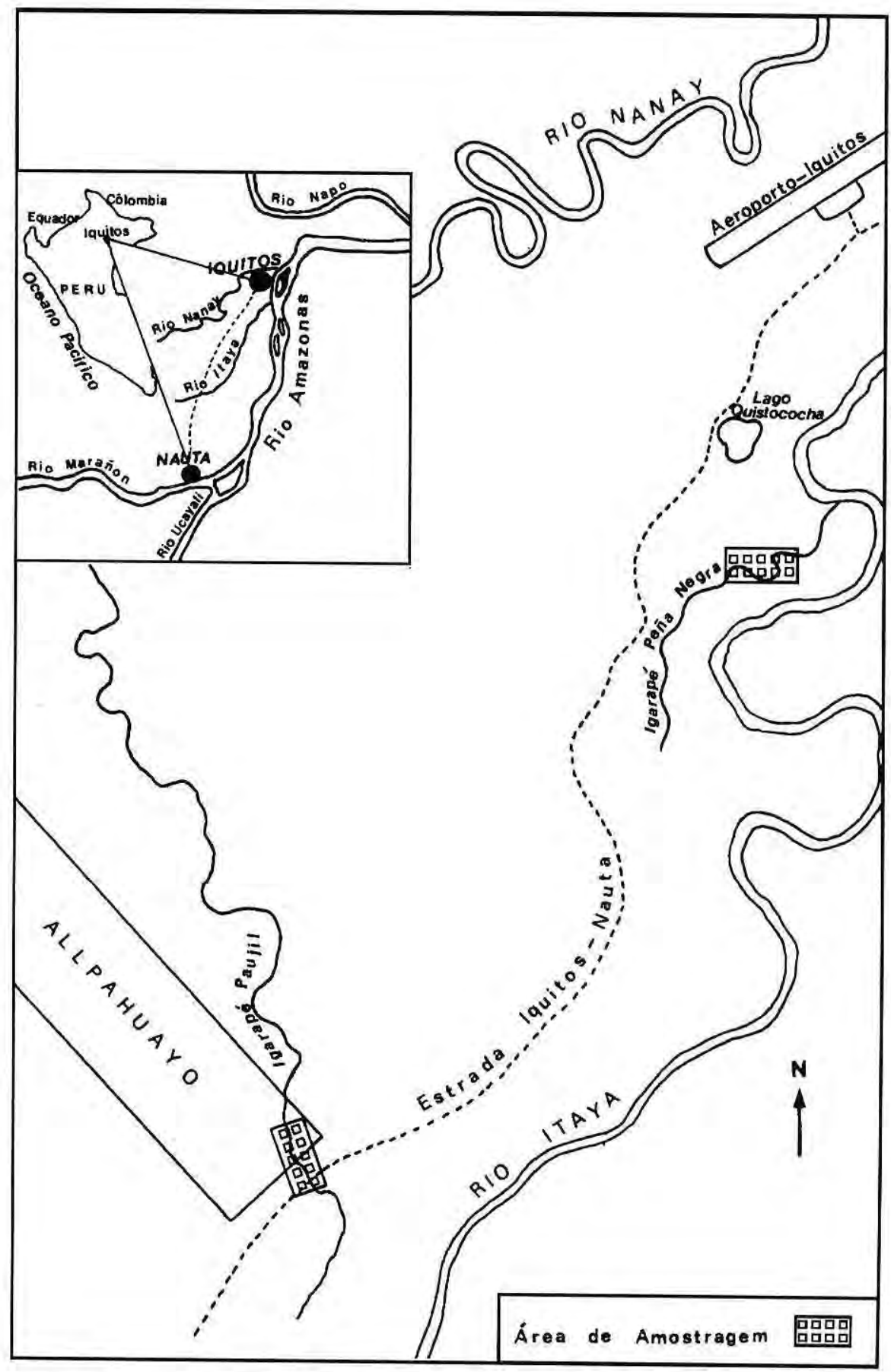

Figura 1. Área de estudo de Macrobrachium brasiliense (Heller, 1862) na estrada Iquitos-Nauta, Loreto-Peru. 
número de ovos nas fềmeas ovadas. A relação entre o peso e o comprimento cefalotorácico foi testada com uma análise de regressão simples. $\mathrm{O}$ volume do ovo foi determinado segundo Medeiros et al. (1994), com a seguinte relação:

$$
\mathrm{V}=(\mathrm{A})^{2} \times 3,14 \times \mathrm{L} / 6
$$

onde: $\mathrm{V}=$ Volume em $\mathrm{mm}^{3}, \mathrm{~A}=$ Diâmetro menor do elipsóide, $\mathrm{L}=$ Diâmetro maior do elipsóide. A relação entre o volume do ovo com o comprimento cefalotorácico e a fecundidade também foi testada mediante análise de regressão simples.

\section{RESULTADOS}

\section{Caracterização sistemática}

$M$. brasiliense caracteriza-se por possuir um rostro reto, com a extremidade distal ligeiramente dirigida para acima, alcançando o final do escafocerito. $\mathrm{O}$ número de dentes na margem superior varia de 8 a 13, com 1 a 4 dentes pós-orbitais, e 2 a 5 na margem inferior. $O$ segundo par de pereiópodos é bem desenvolvido, com os apêndices semelhantes na forma, mas diferentes em tamanho. O cefalotórax dos machos apresentou-se um pouco maior que o das fềmeas (comprimento médio encontrado nos machos $=14,8 \mathrm{~mm}, \pm$ 3,4 ; nas fêmeas $=12,9 \pm 2,0 \mathrm{~mm} ; \mathrm{T}=$ 6,767; g.1. 421; p< 0,0001).

Dimorfismo sexual do segundo par de pereiópodos

Os machos apresentaram pereiópodos maiores e variáveis em tamanho em relação às fêmeas (comprimento médio nos machos $34,77 \pm 15,20 \mathrm{~mm}$; nas fêmeas, 28,05 $\pm 6,85 \mathrm{~mm} ; \mathrm{T}=5,572 ;$ g. 1.421 ; $\mathrm{p}<0,0001$ ). Nas fềmeas, o pereiópodo representa, em média, 2,26 vezes o comprimento do cefalotórax, enquanto que, nos machos, representa, em média, 2,51 vezes o comprimento do cefalotórax. Este valor mostrou uma grande variação no caso dos machos (C.V. $=22 \%)$. Nos machos, o desenvolvimento do pereiópodo esteve mais fortemente relacionado com o desenvolvimento do cefalotórax que nas fêmeas $(\mathrm{p}=0,05)$ (Figs. $2 \mathrm{~A}$ e B).

\section{Proporção de sexos}

Durante todo o periodo de coleta observou-se uma ligeira predominância de fêmeas na população. A porcentagem média, considerando os valores de todas as amostras mensais, foi de $62 \%$ de fêmeas e $38 \%$ de machos, com uma proporção fêmea: macho de 1,7:1. O teste de qui-quadrado $\left(\mathrm{c}^{2}\right)$ mostrou que essa relação, em todos os meses de coleta, com exceção de março e abril, é significativa $\left(X^{2}=169.32 ; n=2604\right.$; $\mathrm{p}=0,05)$.

\section{Período de desova}

Nos dois igarapés estudados, $M$. brasiliense apresentou fêmeas ovadas durante todo o período de coleta (com exceção de março), com um pico reprodutivo de abril a julho, periodo em que a precipitação se encontrava com valores médios (Fig. 3).

\section{Fecundidade}

Do total de fêmeas coletadas, as ovígeras representaram $2,93 \%$, 

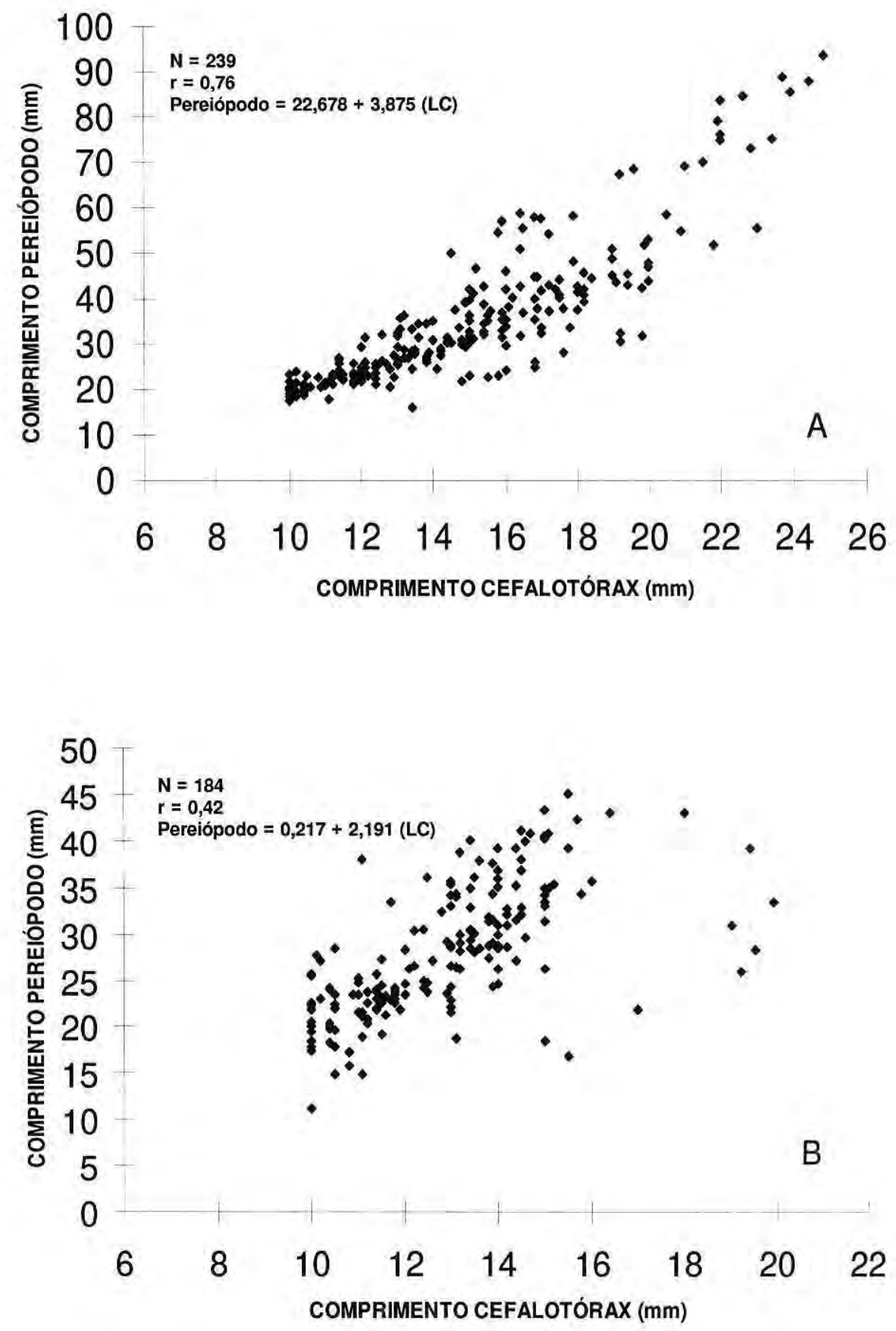

Figura 2. Relação entre o comprimento do segundo pereiópodo e o comprimento do cefalotórax em machos (A) e em fêmeas (B) de Macrobrachium brasiliense (Heller, 1862). 
pesaram de $0,47 \mathrm{~g}$ a $3,14 \mathrm{~g}$ (média $=$ $1,41 \pm 1,17 \mathrm{~g}$ ) e apresentaram comprimento cefalotorácico de 9,0 $\mathrm{mm}$ a $16,9 \mathrm{~mm}$ (média de $11,3 \pm$ $2,97 \mathrm{~mm}$ ). Essas fêmeas carregavam, em média, $82 \pm 52$ ovos. O número de ovos por fêmea oscilou entre 15 e 168 . $O$ peso da postura variou entre 0,02 a $0,74 \mathrm{~g}$.

Do total de fêmeas ovadas capturadas, a maior porcentagem pertence aos intervalos de classe de 13 a $15, \mathrm{~mm}$ que representam $57,8 \%$, (moda $=13$, com $23 \%$ ), decrescendo no intervalo de classe $16(6 \%)$. Não foram capturadas fêmeas ovadas maiores, que permitissem evidenciar a continuidade dessa tendência.

A fecundidade apresentou uma correlação significativa com o comprimento cefalotorácico e o peso (Figs. $4 \mathrm{~A}, \mathrm{~B}$ ), ou seja, à medida que aumenta o comprimento do cefalotórax e o peso da fêmea, aumenta o número de ovos.

\section{Tamanho e volume do ovo}

O comprimento dos ovos variou de 1,5 a $2,2 \mathrm{~mm}$ (média $=1,85 \mathrm{~mm}$ ), com uma largura de 1,1 a $1,7 \mathrm{~mm}$ (média $1,3 \mathrm{~mm}$ ). O volume médio dos ovos foi de $1,88 \pm 0,37 \mathrm{~mm}^{3}$, sendo

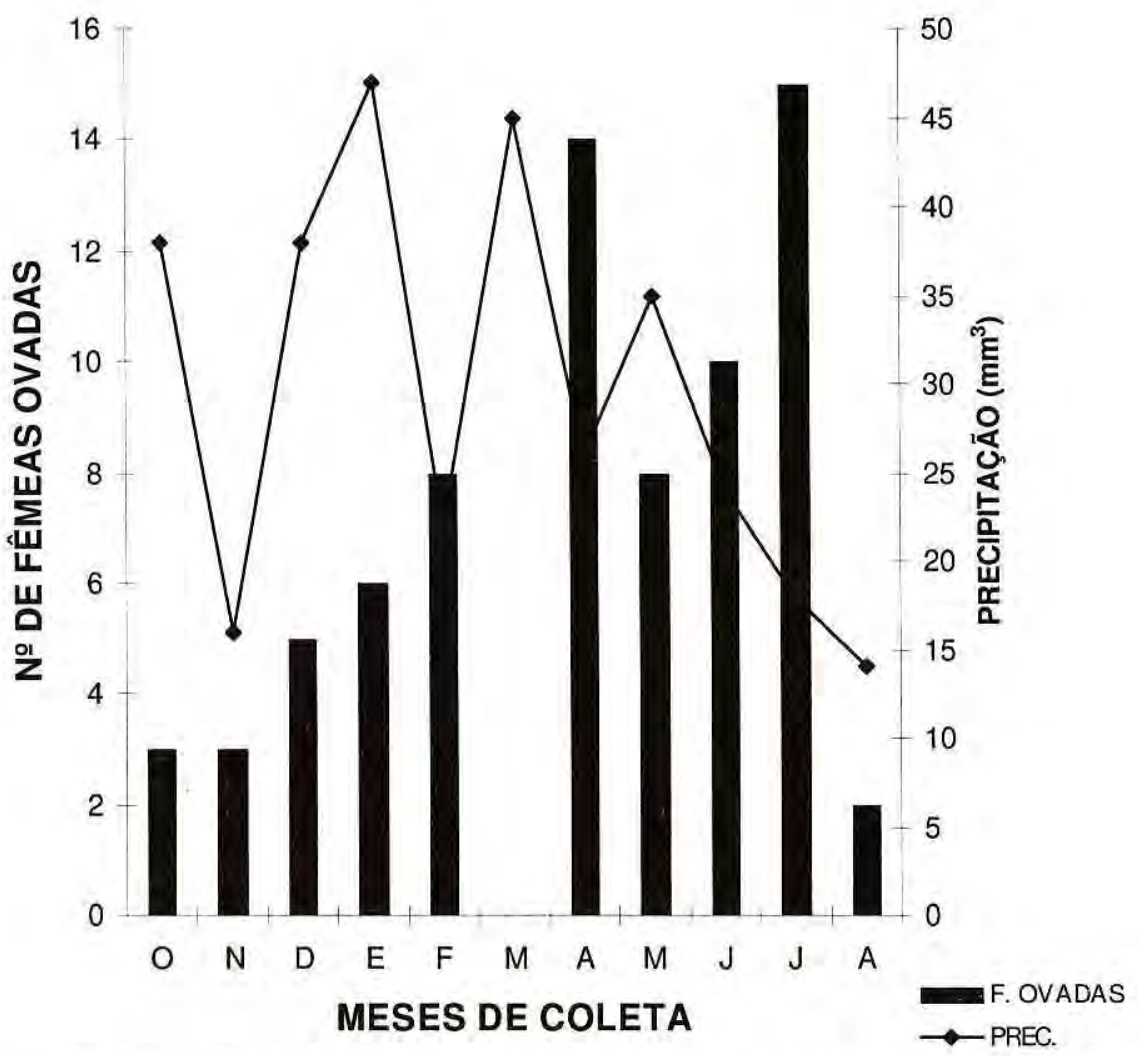

Figura 3. Variação do número de fêmeas ovadas de Macrobrachium brasiliense (Heller, 1862) e precipitação durante o período de coleta. 

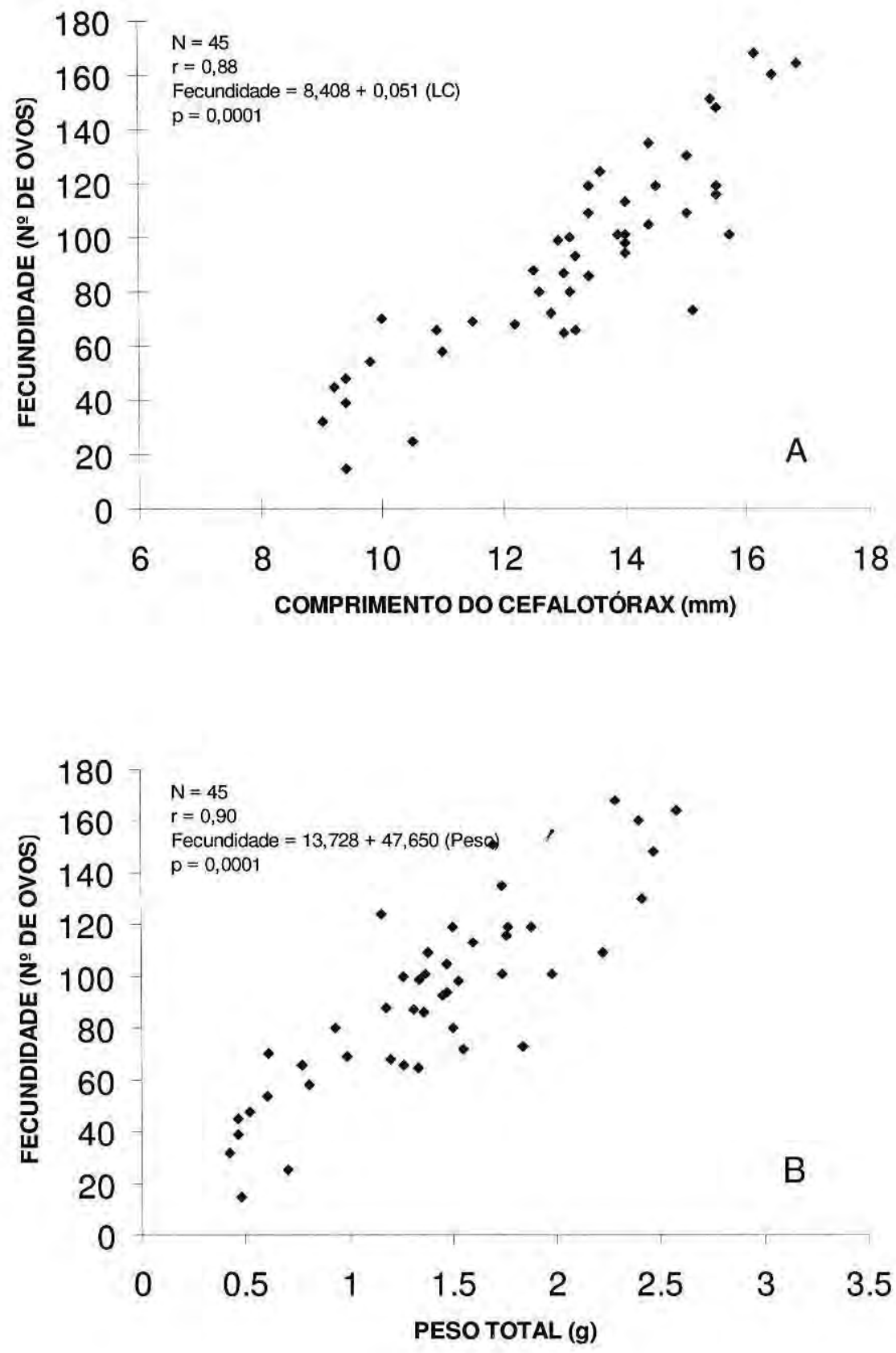

Figura 4. Relação entre a fecundidade, o comprimento do cefalotórax $(\mathrm{A})$ e o peso total $(\mathrm{B})$ de Macrobrachium brasiliense (Heller, 1862). 
que o maior volume médio encontrado por fêmea foi de $2,83 \mathrm{~mm}^{3}$, e o menor, de $1,27 \mathrm{~mm}^{3}$. O volume médio do ovo não apresentou nenhuma correlação com a comprimento cefalotorácico ( $\mathrm{r}$ $=0,0008)$ ou com a fecundidade $(\mathrm{r}=0,0683)$ a um $\mathrm{p}=0,05$.

\section{DISCUSSÃO}

\section{Dimorfismo sexual no segundo par de pereiópodos}

Crescimento heterogêneo associado a fatores intrínsecos, ambientais ou sociais, foi observado em outras espécies aquáticas (Wilbur \& Collins, 1973; Newkink et al., 1977). Ra'ánan \& Cohen (1984) comprovaram que no crescimento em cativeiro de juvenis de $M$. rosenbergii, os fatores sociais controlam mais efetivamente a variação no tamanho de juvenis que os fatores genéticos. Observaram crescimento heterogêneo com duas classes de tamanho de camarões cultivados em grupo e crescimento homogêneo em camarões cultivados em isolamento.

Possivelmente tal fato também poderia ocorrer em ambientes naturais e que a diferença de tamanho no segundo par de pereiópodos entre os sexos de $M$. brasiliense responderia a pressões de grupo, nas quais os machos precisariam desenvolver pereiópodos maiores do que os das fêmeas para defender seu território e seu clã da invasão de outros machos. Isso também explicaria a ampla variação apresentada entre os machos, já que a existência de machos dominantes territorialistas envolveria uma hierarquização dentro de uma determinada área, com machos dominados sob a influência dos dominantes.

\section{Período de desova}

Os camarões de água doce podem apresentar reprodução contínua ou periódica. Nas regiões de latitudes maiores, a estação reprodutiva possa ocorrer por ação de um estímulo primário fotoperiódico, iniciando-se a partir do inverno e com época de eclosão das pós-larvas nos meses de verão (Bond \& Buckup, 1982), enquanto nos trópicos a época de reprodução está, principalmente, relacionada ao regime hidrológico. $\mathrm{Na}$ Amazônia, reprodução contínua foi constatada em $M$. amazonicum, que habita as várzeas dos rios de águas brancas, ricas em nutrientes (Odinetz Collart, 1992) em Macrobrachium inpa (Kensley \& Walker, 1982) $e$ Pseudopalaemon amazonensis (Ramos-Porto, 1979), que habitam pequenos igarapés permanentes de terras mais altas (Kensley \& Walker, 1982; Walker \& Ferreira, 1985). Por outro lado, Palaemonetes carteri (Gordon, 1935), que habita igarapés de áreas baixas próximas aos grandes rios de água preta e que sofrem inundação anual, apresenta reprodução somente no período de enchente das águas (Walker \& Ferreira, 1985; Odinetz Collart \& Enriconi, 1993). No presente estudo, as populações de $M$. brasiliense, que ocorrem nos igarapés de terras altas da estrada Iquitos-Nauta 
apresentaram desova durante o ano todo, mas com maior intensidade no período de abril a julho.

Estes diferentes padrões reprodutivos apresentados por camarões que habitam hidrossistemas diferentes representaria uma resposta adaptativa às condições ambientais. Assim, M. amazonicum, que habita águas brancas e claras ricas em nutrientes, capazes de suportar milhares de larvas planctotróficas, se reproduz durante $o$ ano todo (Odinetz Collart \& Enriconi, 1993). Por sua vez, $M$. brasiliense que habitam igarapés rasos de terra firme, onde o pulso de inundação é muito mais dinâmico e de menor duração, reproduz-se $o$ ano todo, assegurando a presença tanto temporal como espacial das larvas em condições ambientais tão instáveis. Ao contrário, P carteri, que ocorre em igarapés do baixo rio Negro, desova somente durante o período de inundação do rio, o que garantiria a sobrevivência das larvas. A dispersão larval no meio aquático em expansão aumentaria as possibilidades de sobrevivência da espécie, devido à diminuição da competição intra-específica com o aumento dos recursos alimentícios e espaciais. Esse tipo de estratégia reprodutiva é comum em espécies tropicais (Welcomme, 1985; Odinetz Collart \& Enriconi, 1993).

\section{Fecundidade}

A fecundidade (15 e 168 ovos) das fêmeas da população de $M$. brasiliense estudada neste trabalho foi superior à encontrada por Vega (1984), que encontrou fêmeas portando de 63 a 108 ovos. O número de ovos produzidos por uma determinada espécie pode apresentar variações devido às diferenças na idade e no tamanho dos exemplares analisados, às características genéticas das populações e à origem dos animais. Sabe-se que, nos crustáceos, a fecundidade pode se alterar de acordo com as condições fisiológicas das fêmeas, estações do ano, latitude e condições ambientais, bem como com a oferta alimentar ou a temperatura (Jensen, 1958; Coelho et al., 1982; Odinetz Collart \& Magalhães, 1994).

Correlação significativa entre número de ovos e tamanho da fềmea já foi observado em outros camarões amazônicos que apresentam desenvolvimento larval completo e habitam águas brancas, como Macrobrachium surimanicum (Holthuis, 1948) e $M$. amazonicum (Odinetz Collart, 1992; Odinetz Collart \& Magalhães, 1994). Entretanto, em $P$. carteri, que apresentou desenvolvimento larval abreviado e habita igarapés de terra firme pobres em nutrientes, não foi observada nenhuma relação entre $o$ número de ovos e o tamanho da fềmea (Collart \& Enriconi, 1993), indicando que fatores genéticos sejam os principais controladores da fecundidade em espécies que habitam águas ricas em nutrientes. Fatores ecológicos, como a oferta alimentar ou a densidade populacional, poderiam controlar não só o tipo de desenvolvimento larval mas a própria fecundidade nos indivíduos de espécies que habitam águas pobres em nutrientes (Odinetz Collart \& 
Magalhães, 1994). Os resultados obtidos no presente estudo contrapõem-se a esta última afirmação, indicam que a fecundidade estaria influenciada tanto por fatores ecológicos do meio aquático, quanto por fatores genéticos, em espécies que habitam águas pobres em nutrientes.

Na tabela 1, observa-se que, entre os camarões que habitam corpos de águas da Amazônia central, $M$. brasiliense apresenta uma fecundidade muito inferior àquela apresentada por M. amazonicum, cuja fecundidade variou de 289 a 2259 ovos. Entretanto, apresenta uma fecundidade superior a outras espécies do mesmo gênero, como $M$. inpa, M. nattereri (Heller, 1862) e $M$. ferrerai (Kensley \& Walker, 1982).

\section{Tamanho e volume do ovo}

A relação de tamanho do ovo e a distribuição ecológica está bem documentada na literatura (Walker \& Ferreira, 1985; Magalhães \& Walker, 1988; Odinetz Collart \& Rabelo,
1996). Os camarões que habitam a bacia amazônica e que realizam todo seu ciclo reprodutivo independente das águas estuarinas, podem apresentar duas estratégias reprodutivas diferentes. Numa delas, a espécie apresenta alta fecundidade, ovos pequenos e desenvolvimento larval completo. É o caso de $M$. amazonicum, que habita sistemas de águas brancas, ricas em nutrientes, capazes de suportar um número elevado de larvas planctotróficas. $\mathrm{Na}$ outra, a espécie tem baixa fecundidade, ovos grandes e ricos em vitelo e desenvolvimento larval abreviado. Esta estratégia é aquela apresentada pelos camarões que habitam os sistemas de águas pretas e claras, pobres em nutrientes e, por conseguinte, em fitoplâncton (Magalhães \& Walker, 1988; Odinetz Collart \& Magalhães, 1994). M. brasiliense utiliza esta última estratégia, tendo em vista sua baixa fecundidade e o tamanho relativamente grande dos ovos pese ao

Tabela 1. Relação entre o hábitat e a reprodução de cinco espécies de camarões Amazônicos de gênero Macrobrachium.

\begin{tabular}{|c|c|c|c|c|c|}
\hline Espécies & Habitat & $\mathrm{N}^{2}$ de ovos & Tamarho do ovo (mm) & $\mathrm{N}^{0}$ de estádios larvais & Fonte \\
\hline M. amazoriaum & $\begin{array}{c}\text { Sistema de águas brancase } \\
\text { claras. }\end{array}$ & $289-2259$ & $1,06 \times 0,77$ & $10-11$ & Magahăes, 1985 \\
\hline & 1 & & & & \\
\hline M. erreira & $\begin{array}{l}\text { Sistema de águas pretas } \theta \\
\text { igarapés de terra ime. }\end{array}$ & $25-35$ & $3,66 \times 2,44$ & 3 & Magahăes \& Walker, 1988 \\
\hline M. nattereri & $\begin{array}{l}\text { Sisımas de águas pretas e } \\
\text { daras, igarapés de terra irme }\end{array}$ & $34-150$ & $2,58 \times 2,14$ & 3 & $\begin{array}{c}\text { Rooriguez, } 1982 \\
\text { Magaháes \& Waker } 1988\end{array}$ \\
\hline M. inpa & $\begin{array}{l}\text { Sisırnas de águas pretas e } \\
\text { claras, igarapés de terra irme. }\end{array}$ & 6.30 & $1,76 \times 1,31$ & 3 & Wake \& Ferreira, 1985 \\
\hline M. brasiliense & $\begin{array}{l}\text { Sistemas de águas pretas e } \\
\text { claras, igarapés de terra írme. }\end{array}$ & $15-168$ & $2,41 \times 1,91$ & 3 & Presente esudo \\
\hline
\end{tabular}


tamanho das fềmeas adultas. A espécie apresenta desenvolvimento abreviado, com as larvas eclodindo num estado bastante avançado de desenvolvimento, muito parecido a um camarão juvenil (Vega, 1984).

\section{AGRADECIMENTO}

Os autores agradecem aos pesquisadores do INPA: Dr. Célio Magalhães e Dr. Carlos A. Lima pelas críticas e sugestões ao manuscrito. Ao Instituto de Investigaciones de la Amazonía Peruana - IIAP pelo apoio financeiro e pelas bolsas de pesquisas concedidas.

\section{Bibliografia citada}

Bond, G.; Buckup, L. 1982. O ciclo reprodutor de Macrobrachium borrellii (Nobili, 1896) e Macrobrachium potiuna (Muller, 1880) (Crustacea, Decapoda, Palaemonidae) e suas relações com a temperatura. Revista Brasileira de Biologia, 42(3): 473-483.

Coelho, P.A.; Ramos-Porto, M.; Soares, C. M. A. 1982. Biologia e cultivo de camarōes de água doce. In: Série Aqücultura $N$ № 1 . Universidade Federal de Pernambuco, Recife, Pernambuco, Brasil. p.1-53.

Coelho, P.A.; Ramos-Porto, M. 1985. Camarões de água doce do Brasil: distribuição geográfica. Revista brasileira de Zoologia, 2(4): 405-410.

Heller, C. 1862. Beitrage zur naheren kentniss der Macrouren. Sitzungs Berichte der Akademie Wissenschaften in Wiens, 45: 389-426.

Holthuis, L.B. 1952. A geral revision of the Palaemonidae (Crustacea: Decapoda : Natantia) of the Americas, II: the subfamily Palaemonidae. Allan Hancock Foundation Publications, Occasional Paper, 12: 1-79

García-Dávila, C. 1998. Revisão taxônomica dos camarões de água doce (Crustacea: Decapoda: Palaemonidae, Sergestidae) da Amazónia peruana. Dissertação de Mestrado, Instituto Nacional de Pesquisas de Amazônia/Fundação Universidade do Amazonas. Manaus, Amazonas. 62p.

Jensen, J.P. 1958. The relation between body size and number of eggs in marine malacostrakes, Meddel. Danmarks Fish og Hauunder-Sogn. Ser. II, (19): 1-25.

Kensley, B.; Walker I. 1982. Palaemonid shrimps from the Amazon Basin, Brazil (Crustacea: Decapoda: Natantia). Smithsonian Contribuitions to Zoology, 362: 1-18.

López, B.; Pereira, G. 1996. Inventory of the decapod crustaceans of the high and middle Orinoco river delta, Venezuela. Acta Biologica Venezuelica, 16(3): 45-46.

Magalhães, C.; Walker, I. 1988: Larval development and ecological distribution of central amazonian palaemonid shrimps (Decapoda: Caridea). Crustaceana, 55(3): 279-292.

Medeiros, N.; Magalhães, C.; Odinetz Collart O. 1994. Caracterização morfológica e reprodutiva do camarão Macrobrachium inpa (Decapoda, Palaemonidae). In: anais-46 Reunião Anual de SBPC, Universidade Federal do Espirito Santo. p. 826-836.

Montreuil, V.H.; Maco, J.; Tello, S.; Ismiño, R.; Sanches, H. 1990. Cuadro ambiental de la cocha Carocurahuaite y las posibilidades de explotación del camarón Macrobrachium amazonicum. Folia Amazónica, 2: 99-121.

Newkirk, G.F.; Haley, L.E.; Waugh, D.L. Doyle, R. 1977. Genetic of larvae and spat growt rates in the oyster Crassostrea virginica. Marine Biology, 41: 49-52.

Odinetz Collart, O. 1988. Aspectos ecológicos do camarão Macrobrachium amazonicum (Heller, 1962) no baixo Tocantins (PABrasil). Memorias de la Sociedad de Ciencias Naturales La Salle, XLVIII, suplemento: 341-353.

Odinetz Collart, O. 1992. Ecologia e potencial pesqueiro do camarão-canela Macrobrachium amazonicum na bacia 
amazônica In: Ferreira, E.J.; Santos, G.M.; Leão, E.L.M.; Oliveira, L.A. (Eds). Bases Cientificas para Estratégias de Preservação e Desenvolvimento da Amazônia. vol. 2. Instituto Nacional de Pesquisas da Amazônia (INPA). Manaus, Amazonas. p.147-166.

Odinetz Collart, O.; Enriconi, A. 1993. Estratégia reprodutiva e alguns aspectos demográficos do camarão Palaemonetes carteri Gordon, 1935 na Amazônia Central, rio Negro. Acta Amazonica, 23(2-3): 227-243.

Odinetz Collart, O.; Magalhães, C. 1994. Ecological constraints and life history strategies of palaemonid prawns in Amazonia. Verhandlungen der Internationale Vereinigung Limnologie, 25: 2460-2467.

Odinetz Collart, O; Rabelo, H. 1996. Variation in the egg size the fresh-water prawn Macrobrachium amazonicum (Decapoda: Palaemonidae). Journal of Crustacean Biology, 16(4): 684-688.

Pereira, S.G.A. 1993. A description of a new species of Macrobrachium from Perú, and distributional records for Macrobrachium brasiliense (Heller) (Crustacea: Decapoda: Palaemonidae). Proceedings of the Biological Society of Washington, 106(2): 339-345.
Ra'anan, Z; Cohen, D. 1984. The effect of group interaction on the develoment of size distribution in Macrobrachium rosembergii (De Man) juvenile population. Biologique Bulletin de la France et de la Belgique, 166: 22-31.

Vega, P.L.A., 1984. Desenvolvimento larval de Macrobrachium heterochirus (Wiegman, 1836), Macrobrachium amazonicum (Heller, 1862) e Macrobrachium brasiliense (Heller, 1868) (Crustacea, Decapoda, Palaemonidae), em laboratório. Tese de doutorado, Universidade de São Paulo, São Paulo. 255p.

Walker, I,; Ferreira, M.J.N. 1985. On the population dynamics and ecology of the shrimp species (Crustacea, Decapoda, Natantia) in the central Amazonian river TarumaMirim. Oecologia, 66: 264-270.

Welcome, R.L. 1985. River Fisheries, FAO Fisheries Technical Paper 262. FAO Publications, Roma, Italy. 330p.

Wilbur, H.M.; Collins, J.P. 1973. Ecological aspects of amphibian metamorphosis. Science, 182:1305-1314. 